OPEN ACCESS

Edited by:

Carla Maria Pedrosa Ribeiro, University of North Carolina at

Chapel Hill, United States

Reviewed by: Francesco Maione, University of Naples Federico II, Italy Christine Vigeland, University of North Carolina at Chapel Hill, United States

${ }^{*}$ Correspondence: Hua Jin

jinhua0413@gdmu.edu.cn Xinguang Liu xgliu@gdmu.edu.cn

${ }^{t}$ These authors have contributed equally to this work

Specialty section: This article was submitted to Inflammation Pharmacology, a section of the journal Frontiers in Pharmacology

Received: 06 August 2020 Accepted: 12 October 2020 Published: 27 January 2021

Citation:

Jin H, Zhao Z, Lan Q, Zhou H, Mai Z, Wang $Y$, Ding $X$, Zhang W, Pi J, Evans CE and Liu $X$ (2021) Nasal

Delivery of Hesperidin/Chitosan Nanoparticles Suppresses Cytokine Storm Syndrome in a Mouse Model of Acute Lung Injury.

Front. Pharmacol. 11:592238. doi: 10.3389/fphar.2020.592238

\section{Nasal Delivery of Hesperidin/Chitosan Nanoparticles Suppresses Cytokine Storm Syndrome in a Mouse Model of Acute Lung Injury}

\author{
Hua Jin ${ }^{1+\star}$, Zuguo Zhao ${ }^{1 \dagger}$, Qian Lan ${ }^{1}$, Haotong Zhou ${ }^{1}$, Zesen Mai ${ }^{1}$, Yuan Wang ${ }^{1}$, \\ Xiaowen Ding ${ }^{1}$, Wenting Zhang ${ }^{1}$, Jiang $\mathrm{Pi}^{2}$, Colin E. Evans ${ }^{3}$ and Xinguang Liu ${ }^{1 *}$ \\ ${ }^{1}$ College of Pharmacy, Guangdong Provincial Key Laboratory of Medical Molecular Diagnostics, Guangdong Medical University, \\ Dongguan, China, ${ }^{2}$ Department of Microbiology and Immunology, University of Illinois at Chicago, Chicago, IL, United States, \\ ${ }^{3}$ Feinberg School of Medicine, Northwestern University, Chicago, IL, United States
}

The cytokine storm or cytokine storm syndrome (CSS) is associated with high mortality in patients with acute lung injury (ALI) and acute respiratory distress syndrome (ARDS), for example following sepsis or infectious diseases including COVID-19. However, there are no effective treatments for CSS-associated ALI or ALI/ARDS. Thus, there remains an urgent need to develop effective drugs and therapeutic strategies against CSS and ALI/ ARDS. Nasal and inhaled drug delivery methods represent a promising strategy in the treatment of inflammatory lung disease as a result of their ability to improve drug delivery to lungs. Improving the nasal mucosa absorption of poorly water-soluble drugs with poor mucosa bioavailability to a therapeutically effective level is another promising strategy in the fight against ALI/ARDS. Here, chitosan nanoparticles loaded with hesperidin (HPD/NPs) were developed for nasal delivery of the anti-inflammatory HPD compound to inflammatory lungs. In vitro and in vivo, HPD/NPs exhibited enhanced cellular uptake in the inflammatory microenvironment compared with free HPD. In a mouse model of inflammatory lung disease, the HPD/NPs markedly inhibited lung injury as evidenced by reduced inflammatory cytokine levels and suppressed vascular permeability compared with free HPD. Collectively, our study demonstrates that nasal delivery of HPD/NPs suppresses CSS and ALI/ARDS in a murine model of inflammatory lung disease, and that nanoparticlebased treatment strategies with anti-inflammatory effects could be used to reduce CSS and $A L I$ in patients with inflammatory lung injury.

Keywords: cytokine storm syndrome, hesperidin, chitosan nanoparticle, lung inflammation, nasal drug delivery

\section{INTRODUCTION}

Inflammatory lung injury, including sepsis-induced acute lung injury (ALI) and acute respiratory distress syndrome (ARDS), is associated with increased expression of pro-inflammatory cytokines. This so-called cytokine storm syndrome (CSS) is characterized by excessive amounts of proinflammatory cytokines, such as the interleukins (IL), interferons (IFN), and tumor necrosis factors (TNF) (Gupta et al., 2020). CSS is associated with clinical deterioration and high mortality in patients with viral infections, such as COVID-19 (Mehta et al., 2020), SARS (Channappanavar and Perlman, 2017) and influenza. At present, there are no efficient drugs or strategies to treat CSS and CSS-associated ALI/ARDS in severe COVID-19 cases. 
Although corticosteroids have been used to inhibit CSS in COVID-19 patients, such drugs are limited by their side effects (Russell et al., 2020). Furthermore, several antiinflammatory agents have been shown to reduce ALI and inflammation in experimental studies, but have failed in clinical trials of ALI/ ARDS patients (Matthay et al., 2017). Alternative antiinflammatory methods targeting CSS are under development, such as interleukin- 6 antibodies, and have been used to combat COVID-19 (Zhang et al., 2020). Long-term use of antibody treatments, however, could cause chronic immunosuppression. Thus, the development of novel therapies to effectively control and target ALI and CSS is an urgent requirement in the fight against sepsis-induced ALI/ARDS, COVID-19, or other infectious inflammatory lung diseases. One potential limitation of anti-inflammatory agents is the off-target delivery to, and short-term retention of, the agents in lungs. In this work, a nasal nanoparticle (NP)-based drug delivery system was designed to deliver an inflammatory agent (Hesperidin, HPD) to inflammatory lungs with the view to reducing CSS and ALI/ ARDS. HPD is an active flavonoid aglycone (Hemanth Kumar et al., 2017) found in citrus fruits, which has shown no side effects or toxicity in experimental animal studies (Li et al., 2019). Along with its anti-inflammatory properties, HPD exhibits analgesic, anti-carcinogenic, anti-viral (Ciftci et al., 2015), and anticoagulant activities (Roohbakhsh et al., 2015).

Many investigators are aiming to improve the development of drug-loaded NPs and the delivery of drugs to infectious sites via cell- or tissue-specific targeting techniques (Wang et al., 2018). Nasal drug delivery systems can be employed to reduce elimination by the liver, kidneys, and gastrointestinal tract (Sukumar et al., 2019), compared with oral, intraperitoneal, or intravenous delivery routes. However, nasal administration of HPD is limited in vivo because of its poor aqueous solubility and bioavailability. To address this drawback, PLGA or chitosan nanoparticle (NP) delivery systems have been developed to improve the aqueous solubility, safety, and efficacy of pharmacological agents (Jin et al., 2017). Chitosan (CS)-based NPs, for example, can significantly improve mucosal drug delivery, as a result of the electrostatic attraction between positively charged CS chains and negatively charged sialic acid of nasal mucosa (Gholizadeh et al., 2019).

In this work, we synthesized an HPD-loaded PLGA-CS NP delivery system (HPD/NPs) to target HPD to inflammatory lungs via the nasal delivery route, and assessed the capacity of these HPD/NPs to suppress CSS and ALI in a mouse model of endotoxic lung injury.

\section{METHODS AND MATERIALS}

\section{Preparation and Characterization of Hesperidin-Loaded PLGA-Chitosan NPs (HPD/NPs)}

HPD-NPs were synthesized by emulsification and evaporation methods. Briefly, $20 \mathrm{mg}$ HPD and $80 \mathrm{mg}$ PLGA-PEG (1:1 lactide: glycolide, Sigma, USA) were dissolved in $5 \mathrm{ml}$ of dichloromethane and homogenized for 40 seconds to form the oil phase emulsification. The oil phase emulsification was combined with $20 \mathrm{ml}$ PVA ( $1 \% \mathrm{w} / \mathrm{w}$, Sigma, USA) containing of $0.2 \%$ of chitosan and homogenized for another $40 \mathrm{~s}$ to form the second water phase emulsification. This water phase emulsification was added to $100 \mathrm{ml}$ water and stirred for $6 \mathrm{~h}$ to achieve organic reagent evaporation and nanoparticle hardening. Finally, HPD/NPs were harvested by centrifugation at $12,000 \mathrm{rpm}$ for $20 \mathrm{~min}$ and washed 3 times with ultrapure water then lyophilized for $48 \mathrm{~h}$ for storage at $4{ }^{\circ} \mathrm{C}$.

\section{Characterization of NPs}

Size distribution and zeta potential were estimated by dynamic laser scattering using a Zetasizer Nano ZS (Malvern Instruments, UK). NPs were visualized by scanning electron microscopy (Philips Co, Holland). For measuring HPD loading capacity in HPD/NPs, $10 \mathrm{mg}$ lyophilized NPs were dissolved in $1 \mathrm{ml}$ of dichloromethane, and then, the amount of HPD in the solution was determined by high-pressure liquid chromatography (HPLC). HPLC detection was performed using a C18 column $(5 \mu \mathrm{m}, 250 \mathrm{~mm} \times 4.6 \mathrm{~mm})$, whereas the mobile phase, consisting of methanol and $0.1 \%$ acetic acid (88:12) $(\mathrm{v} / \mathrm{v})$, was maintained at a flow rate of $1.0 \mathrm{ml} / \mathrm{min}$. The ultraviolet detector wavelength was $285 \mathrm{~nm}$, and the injection volume was $20 \mu \mathrm{l}$.

\section{Evaluation of HPD Release in vitro}

HPD release from NPs was evaluated in vitro using the dialysis method as previously described (Jin et al., 2017). Briefly, dialysis bags with a molecular weight cut-off of $10,000 \mathrm{Da}$ containing $10 \mathrm{mg}$ of compounds were immersed in a water bath containing $20 \mathrm{ml}$ of PBS ( $\mathrm{pH} \mathrm{7.4)}$ at $37^{\circ} \mathrm{C}$. At indicated times, $1 \mathrm{ml}$ of receiving buffer was withdrawn and replaced with $1 \mathrm{ml}$ of PBS. HPD release from dialysis bag into the water bath was determined by ultraviolet spectrophotometry (Agilent 8,453, Agilent Technologies, USA) at $285 \mathrm{~nm}$.

\section{Cell Culture}

Mouse macrophage RAW264.7 cells and human umbilical vein endothelial cells (HUVECs, Lonza) were cultured in DMEM (Gibco) with $10 \%$ fetal bovine serum (Gibco) containing $100 \mu \mathrm{g} / \mathrm{ml}$ streptomycin and $100 \mathrm{IU} / \mathrm{ml}$ penicillin. Cells were sub-cultured twice/week and incubated in a humidified incubator (Thermo) at $5 \% \mathrm{CO} 2$ and $37^{\circ} \mathrm{C}$.

\section{Cell Viability Assay}

RAW264.7 cells or HUVECs were cultured in 96-well plates for $24 \mathrm{~h}$, then exposed to $10 \mu \mathrm{g} / \mathrm{ml} \mathrm{HPD}$ or HPD/NPs or vehicle (0.02\% DMSO) for $3 \mathrm{~h}$, followed by activation with $1 \mu \mathrm{g} / \mathrm{ml}$ of LPS for $24 \mathrm{~h}$. Cell viability was assessed using the Cell Counting Kit assay (Beyotime Institute of Biotechnology, Nanjing, China) according to the manufacturer's instructions. All experiments were performed three times.

\section{Endothelial Permeability Assay}

The endothelial permeability assay was performed as described (Monfoulet et al., 2017) with the following modifications. In brief, 
HUVECs were seeded onto Corning Transwell filters for $24 \mathrm{~h}$ in medium with $0.2 \%$ FBS then exposed to $10 \mu \mathrm{g} / \mathrm{ml} \mathrm{HPD}$ or HPD/NPs or vehicle $(0.02 \%$ DMSO) for $3 \mathrm{~h}$, followed by activation with $1 \mu \mathrm{g} / \mathrm{ml}$ of LPS for $24 \mathrm{~h}$. Permeability of the endothelium was evaluated by assessing the passage of FITC-dextran $(40 \mathrm{kDa})$ through endothelial monolayer. One hundred microliters of FITC-dextran were added to the upper chamber and allowed to equilibrate for $1 \mathrm{~h}$, after which FITC fluorescence (excitation $488 \mathrm{~nm}$; emission $525 \mathrm{~nm}$ ) in the lower chamber was measured using a microplate reader. Three independent experiments were performed.

\section{Immunofluorescence Staining}

F-actin expression was evaluated by staining with phalloidin FITC. In brief, HUVECs were cultured on coverslips, then fixed with $4 \%$ paraformaldehyde for $30 \mathrm{~min}$ and incubated with $1 \mathrm{mM}$ phalloidin-FITC for $60 \mathrm{~min}$ in the dark at room temperature, and then washed twice with PBS. Cytoskeleton organization was imaged with a laser scanning confocal microscope (LCM 880, Carl Zeiss, Germany). Fluorescence was measured by flow cytometer at excitation wavelength $488 \mathrm{~nm}$, emission wavelength $530 \mathrm{~nm}$ to quantitatively elucidate the alterations of cytoskeleton proteins.

HUVECs cultured on coverslips were fixed with $4 \%$ paraformaldehyde and stained with primary anti VE-cadherin (1:1,000; Sigma-Aldrich) and fluorescence-conjugated secondary antibodies (1:500; Sigma-Aldrich). Nuclei were counterstained with DAPI (DAPI Fluoromount-GTM, thermofisher). Cells were imaged with LCM 880.

\section{In vivo Studies \\ Mice}

C57BL/6 mice were used throughout at 12-14 weeks of age. The experimental protocols were conducted according to National Institutes of Health guidelines on the use of laboratory animals. The animal care and study protocols were approved by the Institutional Animal Care and Use Committee of Guangdong Medical University (GDY2002094).

\section{Mouse Model of Acute Lung Injury}

Lipopolysaccharide (LPS, E. coli 055:B5, Santa Cruz), a component of the cell wall of Gram-negative bacterium, was dissolved in PBS. To induce sepsis, LPS was administered intraperitoneally to mice at $3.5 \mathrm{mg} / \mathrm{kg}$ body weight in $100 \mu \mathrm{L}$ PBS. HPD or HPD/NPs was nasally administrated at $3 \mathrm{~h}$-post LPS challenge, and the animals were sacrificed to collect samples at $24 \mathrm{~h}$ post-LPS challenge.

\section{Histology}

Lung tissues were fixed and processed for H\&E staining. Briefly, lung tissues were fixed by $5 \mathrm{~min}$ instillation of $10 \%$ PBSbuffered formalin through trachea catheterization at a transpulmonary pressure of $15 \mathrm{~cm} \mathrm{H}_{2} \mathrm{O}$, and then overnight at $4^{\circ} \mathrm{C}$ with agitation. After paraffin processing, the tissues were cut into $5 \mu \mathrm{m}$ sections and stained with $\mathrm{H} \& \mathrm{E}$ for histological analysis.

\section{Assessment of Lung Vascular Permeability}

The Evans blue-conjugated albumin (EBA) extravasation assay was used to assess pulmonary vascular permeability (Huang et al., 2016). Briefly, EBA (20 mg/kg) was injected retroorbitally at $45 \mathrm{~min}$ before sacrifice and lung collection following perfusion free of blood with PBS. The extravasated EBA in lung homogenates was expressed as $\mu \mathrm{g}$ of EBA per $g$ of lung.

Total protein levels in bronchiolar alveolar lavage fluid (BALF) were measured via bicinchoninacid-assay (BCA) according to the manufacturer's instructions (Pierce BCA Protein Assay, Thermo Scientific, USA).

\section{Immunohistochemistry}

The Lung sections were incubated overnight at $4{ }^{\circ} \mathrm{C}$ in a humidified chamber with anti-caspase 1 (Proteintech: 22915-1-AP) or anti- IL-1 $\beta$ antibodies (Abcam: ab9722) diluted 1:500 in PBS containing $1 \%$ BSA. Primary antibodies were incubated at $4{ }^{\circ} \mathrm{C}$ overnight followed by secondary antibodies for $1 \mathrm{~h}$ at $37^{\circ} \mathrm{C}$. Proteins were visualized using the $\mathrm{DAB}$ chromogen kit (ZSGB-BIO, Beijing, China). Finally, the lung sections were counterstained with hematoxylin.

\section{Statistical Analysis}

Results are expressed as mean \pm SD. Statistical significance was determined by 1-way ANOVA with a Games-Howell post hoc analysis for multiple-group comparisons. Two-group comparisons were analyzed by the 2-tailed unpaired Student $t$-test.

\section{RESULTS}

\section{Preparation and Characterization of HPD/NPs}

To improve the water solubility and bioavailability of HPD, biodegradable polymer PLGA was employed to encapsulate HPD to form soluble NP carriers, and chitosan was employed to modulate the surface zeta potential of the NPs to positive (Bruinsmann et al., 2019). After formulation of the HPD/NPs, the particle size, zetapotential, morphology, entrapment efficiency, and HPD release were determined by dynamic laser light scattering (DLS), scanning electronic microscopy (SEM) and HPLC, respectively. The size distribution of HPD/ NPs was approximately $200 \mathrm{~nm}$ (Figures $\mathbf{1 A}$,B). The surface charge (zeta potential) was $+22 \mathrm{mV}$ (Figure 1C). The loading rate of HPD into the NPs was $8.1 \%$, and encapsulation rate was $81.02 \%$. The HPD/NP diameter did not change significantly for up to 4 weeks at $37^{\circ} \mathrm{C}$ (Figure 1D). The release of HPD from the HPD/NPs occurred steadily in the first $12 \mathrm{~h}$, with $\sim 75 \%$ and $\sim 90 \%$ of the HPD being released by 12 and $24 \mathrm{~h}$, respectively (Figure 1E). Notably, free NPs had a similar appearance and diameter to HPD/NPs (Supplementary Figure S1A). 

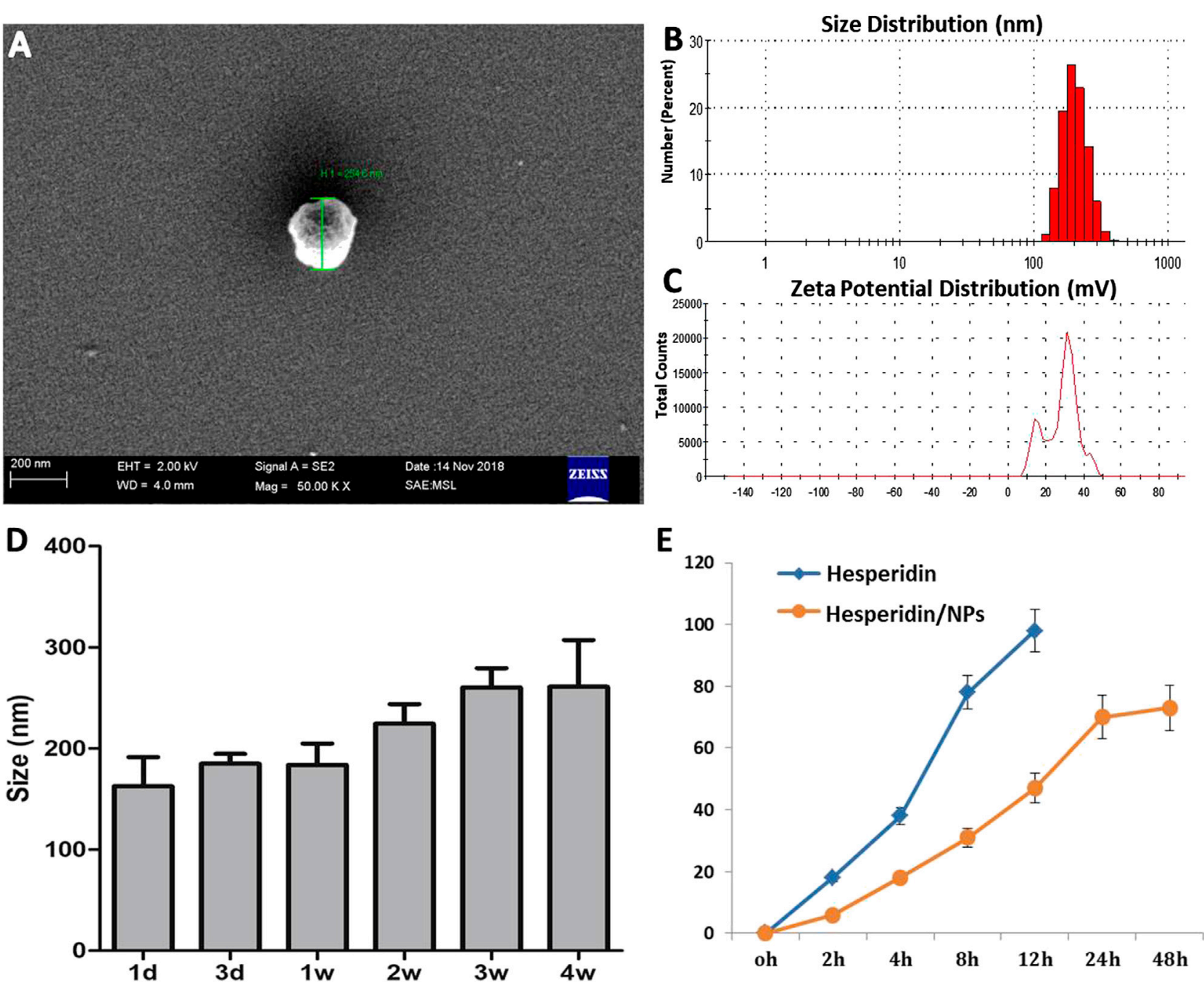

FIGURE 1 | Characterization of HPD/NPs. (A) Scanning electron microscopy (SEM) image of an HPD/NP in suspension. (B) Mean size and (C) zeta potentials of HPD/NPs. (D) HPD/NP size over time ( $n=4$ /group). (E) In vitro release of HPD from HPD/NPs in PBS $(0.01 \mathrm{M}, \mathrm{PH}=7.4, n=4 / g r o u p)$.

\section{In vitro Anti-Cytokine Storm Syndrome Activity of HPD and HPD/NPs}

Figure $2 \mathrm{~A}$ showed that 10 or $50 \mu \mathrm{g} / \mathrm{ml}$ of $\mathrm{HPD}$ or $\mathrm{HPD} / \mathrm{NPs}$ did not induce toxic effects on RAW264.7 cells, while $100 \mu \mathrm{g} /$ $\mathrm{ml}$ of HPD or HPD/NPs caused decreases in cell viability. Therefore, $10 \mu \mathrm{g} / \mathrm{ml}$ concentrations of HPD or HPD/NPs were selected as the dose in the following in vitro and in vivo experiments. Next, RAW264.7 cells were treated with LPS $(1 \mu \mathrm{g} / \mathrm{ml})$ for $24 \mathrm{~h}$. Figure 2B showed that the decreases in cell viability resulting from LPS exposure could be restored by pre-treatment with $10 \mu \mathrm{g} / \mathrm{ml}$ of HPD/NPs, demonstrating that HPD/NPs can protect against LPS-induced cell death in vitro. Figures $2 \mathrm{C}, \mathbf{D}$ indicate that $10 \mu \mathrm{g} / \mathrm{ml}$ of HPD/NPs can significantly suppress the level of inflammation cytokines (NO and IL-6) in LPS-treated RAW264.7 cells. Notably, $2 \%$ DMSO aggravated the inflammatory profile of the RAW264.7 cells, which implied that the DMSO-free delivery of water-soluble HPD/NPs could decrease the side effects of DMSO-based drug delivery. We also found that free NPs did not alter LPS-induced cell viability vs. vehicletreated cells (Supplementary Figure S1B).

\section{HPD/NPs Preserved Endothelial Permeability in vitro}

To delineate the effects of HPD and HPD/NPs on regulating endothelial barrier function, we employed a Transwell system to quantify changes in the integrity of endothelial junctions activated by LPS. HUVECs were plated at $>90 \%$ confluence on Transwell filters to form cell-to-cell contacts and intact monolayers before treatment with compounds and/or LPS. Upon addition of PBS, HPD or HPD/NPs for $2 \mathrm{~h}$, followed by activation with LPS for $24 \mathrm{~h}$, the PBS-treated group exhibited significantly increased permeability compared with the LPS-free basal group, indicative of decreased endothelial barrier function. 


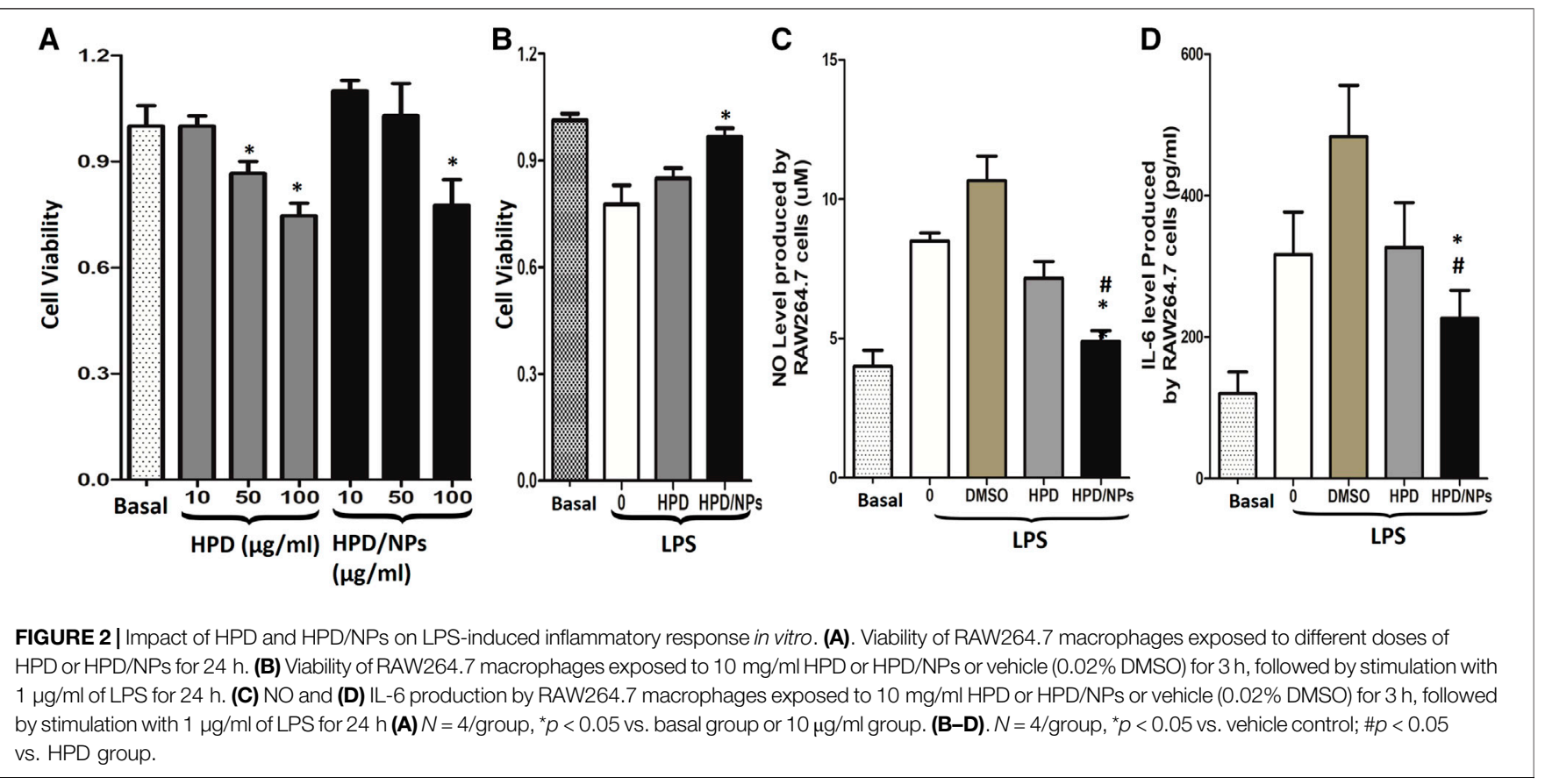

A

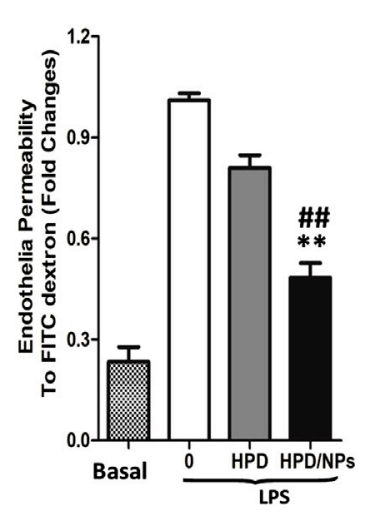

B

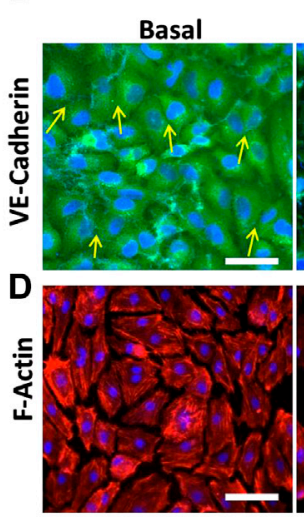

LPS
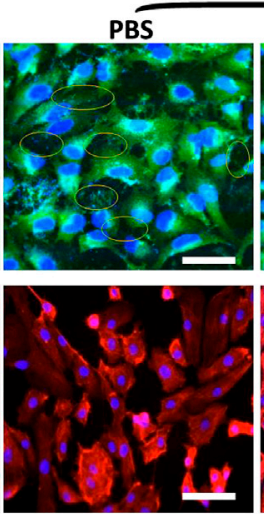

HPD

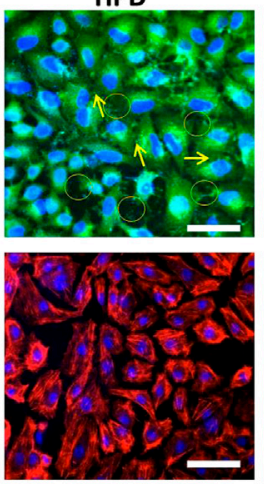

C

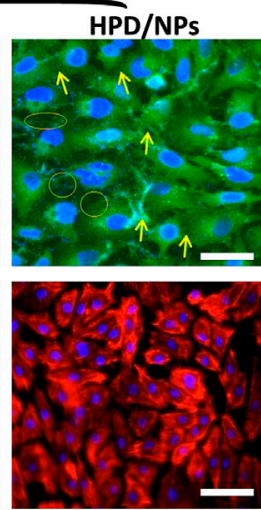

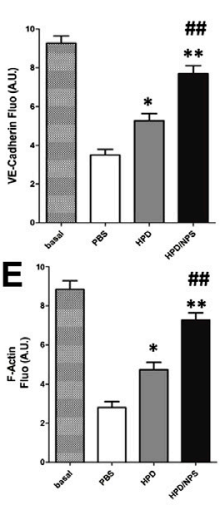

FIGURE 3 I Impact of HPD and HPD/NPs on LPS-induced endothelial integrity in vitro. (A) Permeability of the HUVEC monolayer to FITC-dextran (40 kDa) after $3 \mathrm{~h}$ exposure to PBS or HPD or HPD/NPs $(10 \mu \mathrm{g} / \mathrm{ml})$ followed by $24 \mathrm{~h} \mathrm{LPS} \mathrm{exposure.} \mathrm{Data} \mathrm{expressed} \mathrm{as} \mathrm{a} \mathrm{percentage} \mathrm{of} \mathrm{PBS}+\mathrm{LPS}$ treatment group $\left(n=4 / g r o u p,{ }^{\star \star} p<\right.$ 0.001 vs. vehicle control group; \#\#p < 0.001 vs. HPD group). (B) Representative images and (C) quantification of immunostaining for VE-cadherin (green) in HUVEC monolayers Arrows indicate VE-cadherin-positive cell junctions; circles indicate weak or absent VE-cadherin signal at cell junction. (D) Representative images and (E) quantification of F-actin (red) immunostaining of HUVECs after $3 \mathrm{~h}$ exposure to PBS or HPD or HPD/NPs followed by $24 \mathrm{~h}$ LPS (3.5 mg/kg). Nuclei were counterstained with DAPI (blue). Scale bar, $50 \mu \mathrm{m}{ }^{*} p<0.05$ and ${ }^{* *} p<0.01$ vs. PBS group; \#\#p $<0.01$ vs. HPD group. A.U., arbitrary units.

Figure 3A shows that stimulation of HUVECs by LPS resulted in an approximately 5 -fold increase in endothelial permeability compared to the basal group. This increase in permeability was inhibited by $25 \%$ and $58 \%$ following pre-incubation with $10 \mu \mathrm{g} /$ $\mathrm{ml}$ of HPD and HPD/NPs, respectively. Interestingly, there was no significant change in endothelial permeability in the cells treated with $10 \mu \mathrm{g} / \mathrm{ml} \mathrm{HPD/NPs} \mathrm{compared} \mathrm{with} \mathrm{the} \mathrm{basal} \mathrm{group,}$ indicative of restored endothelial barrier function. To assess the morphological properties of the HUVEC monolayers, VEcadherin and F-actin expression was observed by immunofluorescence microscopy. Figure 3B,E shows that LPS induced EC shrinkage and decreased expression of VEcadherin and F-actin. Excitingly, $10 \mu \mathrm{g} / \mathrm{ml}$ of HPD/NPs could restore normal EC shape and reverse the reduced expression of VE-cadherin and F-actin. Treatment with $10 \mu \mathrm{g} / \mathrm{ml}$ of free HPD, however, failed to decrease the LPS-induced increases in HUVEC permeability or restore VE-cadherin and F-actin expression. These studies showed that HPD/NPs can be used to alleviate LPS-induced increases in endothelial permeability and morphology disruption. 

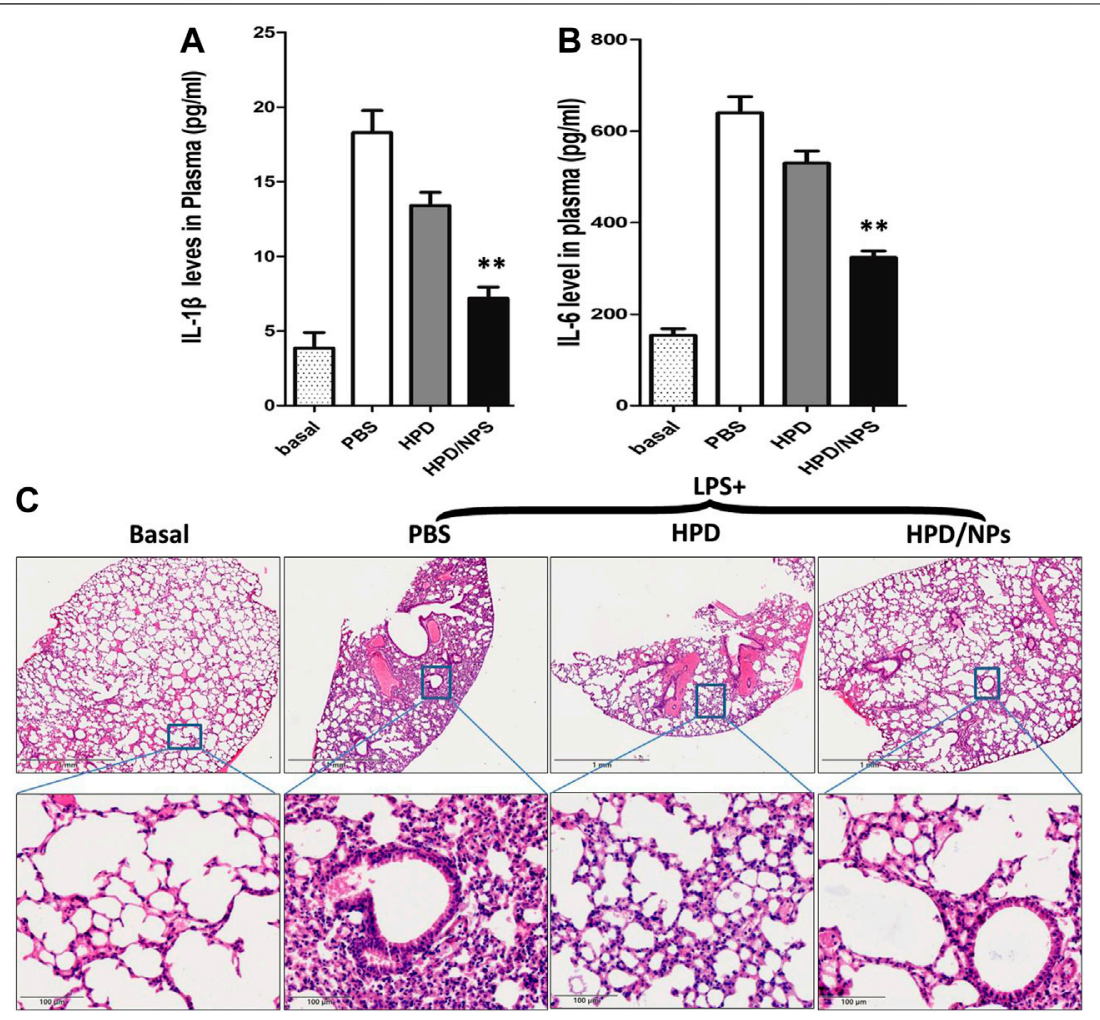

FIGURE 4 | Impact of HPD and HPD/NPs on LPS-induced inflammation in mice. At $3 \mathrm{~h}$ postLPS, PBS (vehicle), HPD, or HPD/NPs were nasally administered to mice. Lung tissues were collected at $24 \mathrm{~h}$ post-LPS challenge. (A) Expression levels of IL-1 $\beta$ and (B) IL-6 in mouse plasma at $24 \mathrm{~h}$ post-LPS challenge $\left(n=4 / \mathrm{group}\right.$; ${ }^{* *} p<$ 0.001 vs. PBS vehicle and vs. HPD). (C) Representative micrographs of H\&E stained lung tissue cross-sections at $24 \mathrm{~h}$ post-LPS challenge. Scale bar, $1 \mathrm{~mm}$ (upper row) or $100 \mu \mathrm{m}$ (lower row).

\section{In vivo Inhibition of Lipopolysaccharide- Induced Cytokine Storm by HPD/NPs}

The effects of HPD or HPD/NPs were further examined in vivo in a mouse sepsis model. LPS injection is commonly used to induce ALI (Lei et al., 2018) and CSS (Du et al., 2018). First, we confirmed that treatment of LPS mice with blank NPs did not alter the level of lung injury vs. LPS mice, as shown by absence of change in BALF protein concentration (Supplementary Figure S1C). Figures 4A,B demonstrates that IL- $1 \beta$ and IL-6 levels in peripheral blood promptly ascended at $24 \mathrm{~h}$ after intraperitoneal injection of LPS.

Treatment with $10 \mathrm{mg} / \mathrm{kg}$ of HPD/NPs efficiently depressed the LPS-induced increases in these inflammatory factors over this short period of time. Moreover, pro-inflammation cytokines such as TNF- $\alpha$ and IL-17 play crucial roles in lung inflammation, so we also determined the levels of such cytokines in BALF. As shown in Supplementary Figure S2A, B, treatment with HPD/NPs inhibited the LPS-induced increases in these inflammatory cytokines. In addition to pro-inflammatory cytokines, the LPSinduced expression of NO (Supplementary Figure S2C) was also decreased by HPD/NPs treatment. Treatment with blank NPs, however, had no discernable effects on inflammatory cytokine levels or NO production in LPS-treated mice, which implied that
HPD acts as the anti-inflammatory agent, while the chitosan NPs act as passive drug carriers. Consistent with these findings, $\mathrm{H} \& \mathrm{E}$ staining showed inflammatory cell infiltration in the lungs of LPS-treated mice (Figure 4C), while treatment with HPD/NPs effectively alleviated the infiltration of inflammatory cells.

\section{HPD/NPs Reduce Lung Injury and Vascular Permeability}

We next investigated the impact of the HPD/NPs on LPS-induced lung injury. We determined alterations in vascular permeability by assessing pulmonary transvascular flux of Evans blue dyeconjugated albumin (EBA) (Huang et al., 2016), bronchiolaralveolar lavage (BAL) protein and wet/dry ratio of lungs. Figure 5A demonstrates the experimental scheme of the EBA assay. Figure 5B shows representative images of lungs extracted from EBA-injected-mice. As expected, LPS treatment resulted in increases in EBA flux, BAL protein, and wet/dry ratio at $24 \mathrm{~h}$ post-LPS compared to basal controls (Figures 5C-E). In mice receiving $\mathrm{HPD}$ at $3 \mathrm{~h}$ post-LPS, there were no discernable improvements in wet/dry ratio or BAL protein at $24 \mathrm{~h}$ postLPS compared to PBS treatment. Mice receiving HPD/NPs, however, showed decreased levels of LPS-induced vascular permeability (EBA flux and BAL protein), and edema (wet/dry ratio). These data demonstrated that nasally administered HPD/ 

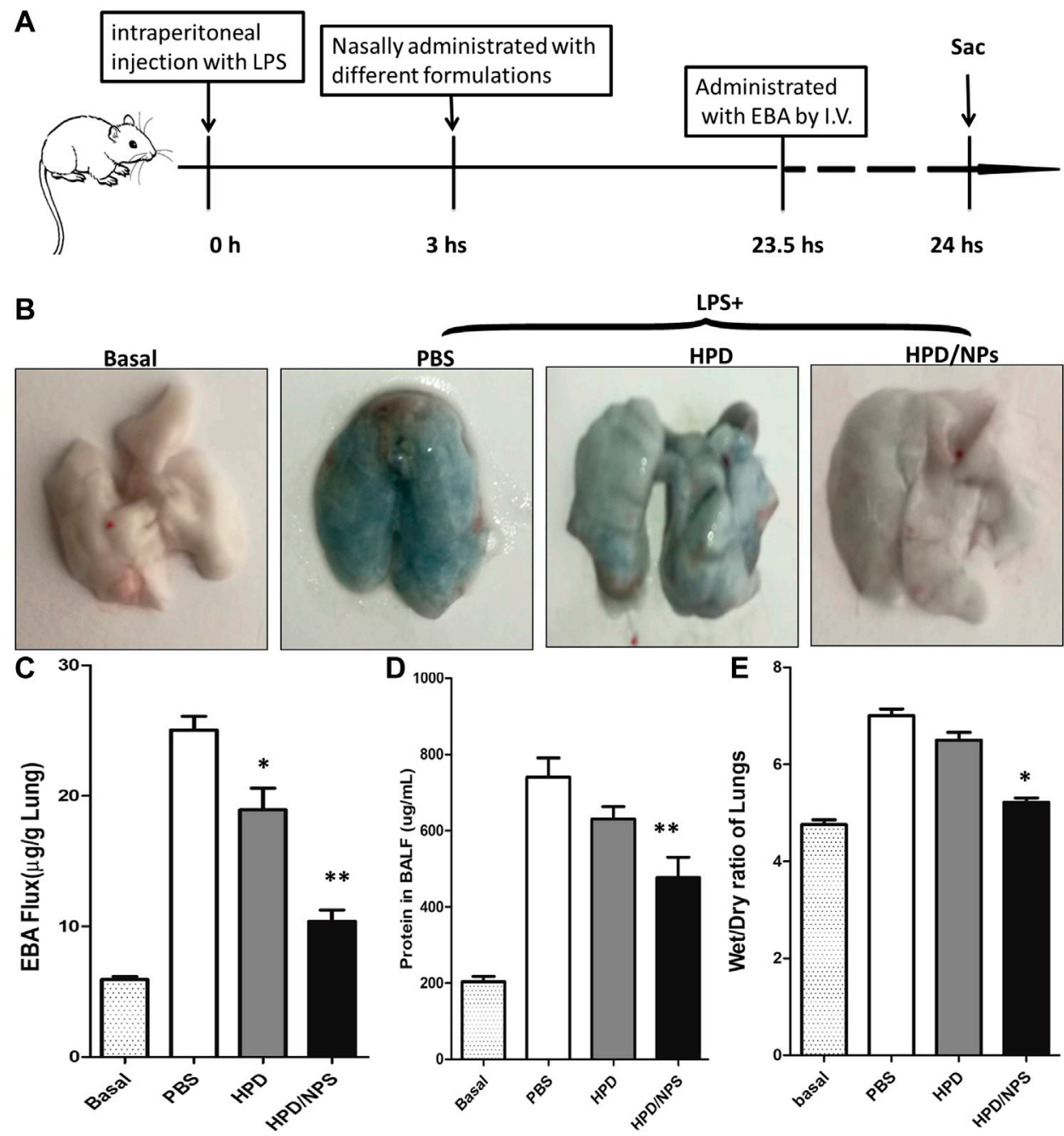

FIGURE 5 | Impact of HPD and HPD/NPS on LPS-induced ALI in mice. At 3 h post-LPS, PBS (vehicle), HPD, or HPD/NPs were nasally administered to mice. Lung tissues were collected at $24 \mathrm{~h}$ post-LPS challenge. (A) EBA assay schematic. (B) Representative images of murine lung tissues after EBA-injection. (C) EBA flux, (D) BAL protein, and (E) wet/dry weight ratio. (C-E) $\mathrm{N}=5$; ${ }^{*} p<0.05$ and ${ }^{* *} p<0.001$ vs. PBS group.

NPs result in targeted delivery of HPD to the inflammatory lungs and inhibition of lung injury.

\section{HPD/NPs Suppress Pyroptosis in Septic Mouse Lungs}

To investigate whether cell pyroptosis (Bergsbaken et al., 2009) was involved in the pathogenesis of ALI in LPS-treated mice and to explore the possible inhibition of LPS-induced pyroptosis by HPD/NPs, immunohistochemical staining was performed to detect the expression levels of IL-1 $\beta$ and caspase 1 in the lung of septic mice (Figure 6). The septic lung tissues in the PBStreated group showed significantly increased expressions of IL- $1 \beta$ and caspase 1 compared with mice in the basal group. Excitingly, the expression of IL-1 $\beta$ and caspase 1 decreased significantly following treatment of LPS mice with HPD/NPs. The inhibitory effect of HPD on pyroptosis was once more less than that of
HPD/NPs, again suggesting that these modified NPs can be used to improve drug delivery and treatment efficacy in inflammatory lung.

\section{DISCUSSION}

ALI following sepsis or infection with SARS-CoV, MERS-CoV, or SARS-CoV-2, represent major healthcare and financial problems worldwide (Su et al., 2016). Despite the threats to human survival and well-being, there are still no effective therapeutic drugs against ALI/ARDS. Additionally, human studies have shown that patients with severe COVID-19 also demonstrate CSS (He et al., 2020). Thus, the develop of novel drugs or treatment strategies against ALI/ARDS and/or CSS is of upmost importance. $\mathrm{Wu}$ et al. (Wu et al., 2020) employed computational methods to identify therapeutic targets for 


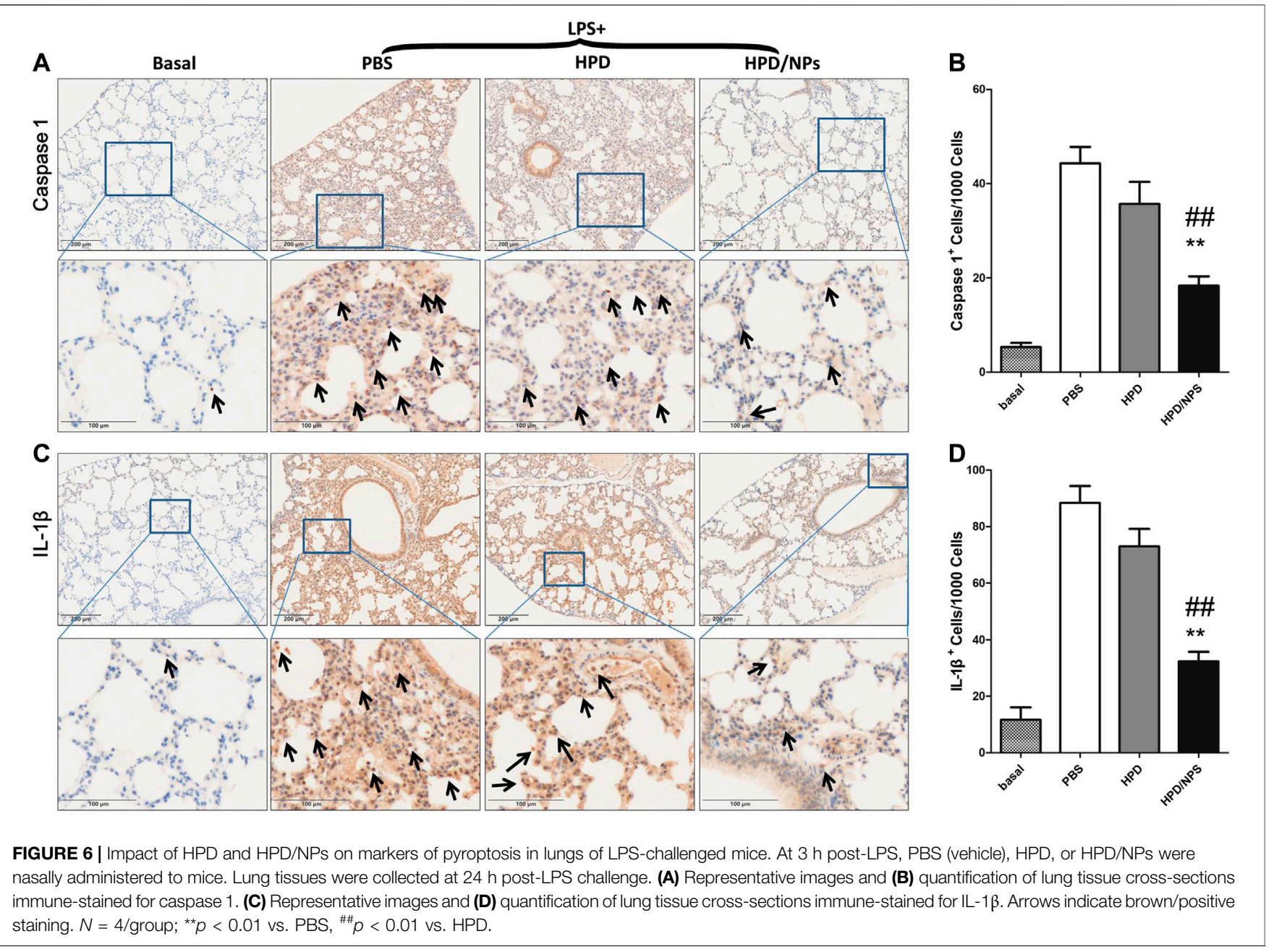

COVID-19 and discovered that HPD could be used as a potential anti- COVID-19 drug. Although this anti-inflammatory and antiviral agent (Lin et al., 2005) can be easily extracted from Isatis indigotica roots and phenolic Chinese herbs, and was extensively used for the prevention of SARS in China, the poor water solubility and bioavailability of HPD limit its efficiency, especially when delivery intranasally.

Herein, we designed a HPD/NP delivery system to target inflammatory lung tissue and reduce CSS. To improve the adsorption rate of the HPD/NPs by nasal mucosa, chitosan was added to the surface of the PLGA NPs (Garg et al., 2019). The zeta potential of the HPD/NPs was $+22 \mathrm{mV}$, suggestive of good adsorption of HPD/NPs into nasal mucosa. However, the impact of different types of NP on nasal mucosal absorption per se should be assessed in future studies.

In vitro, we identified $10 \mu \mathrm{g} / \mathrm{ml}$ as a dose of HPD/NPs that does not induce cytotoxicity in RAW264.7 cells, while $50 \mu \mathrm{g} / \mathrm{ml}$ or $100 \mu \mathrm{g} / \mathrm{ml}$ of both HPD and HPD/NPs did decrease cell viability. Thus, the dose of $10 \mu \mathrm{g} / \mathrm{ml}$ was selected to be assessed in the in vitro and in vivo experiments. While both HPD and HPD/NPs could significantly inhibit the production of inflammatory cytokines (NO and IL-6) in vitro, this inhibition was greater when cells were treated with HPD/NPs compared with HPD alone. In vivo, $10 \mathrm{mg} / \mathrm{kg}$ of $\mathrm{HPD} / \mathrm{NPs}$ but not free HPD significantly suppressed CSS. These studies suggest that the anti-inflammatory impact of HPD is enhanced by delivery in chitosan NPs. This delivery system also alleviated the need for DMSO as a solubility agent in vivo, which could damage nasal mucosa when administrated nasally. Furthermore, excessive amounts of NO can promote cytokine and matrix metalloproteinase production, mitochondrial dysfunction, and cell apoptosis, which aggravates inflammation and tissue injury (Wu et al., 2018). The HPD/NPs significantly inhibited NO release in LPS-challenged mice, implying that nasal delivery of HPD/NPs can enhance the anti-CSS effects of HPD through reductions in multiple different cytokines and signaling molecules.

To determine the impact of HPD/NPs on CSS-associated ALI, we next investigated the effects of HPD/NPs on endothelial barrier function, which plays a vital role in lung inflammation and ALI We showed that the damaged integrity of endothelial monolayers following LPS treatment could be restored by HPD/NP treatment in vitro. Consistent with these findings, we also showed that nasal HPD/NPs significantly attenuated LPS- 
induced increases in ALI in mice, as shown by reductions in vascular permeability, CSS, infiltration of leukocytes, and presence of protein-rich liquid in pulmonary alveoli. Importantly, we showed that the NPs alone did not alter inflammatory cytokine levels or NO production in LPS-treated mice, suggesting that HPD/ NPs but not the NPs alone were active pharmacological agents against CSS-associated ALI. Pyroptosis is involved in the development of inflammatory lung diseases such as ALI/ARDS, and we demonstrated that the HPD/NPs inhibited pulmonary cell pyroptosis markers in LPS-treated mice. These findings together support the possibility that HPD/NPs reduce CSS-associated ALI through decreases in cytokine release, vascular permeability, and cell pyroptosis, and have potential for the development of novel treatment strategies for sepsis and CSS in infectious diseases.

It is worth mentioning that the HPD dose that had protective effects on CSS-induced lung injury in our study is 5-fold lower than HPD dose in free form used to reduce smoke-induced lung inflammation in a previous study (Yu et al., 2019). Previous experimental studies have shown that treatment efficacy can be improved through delivery strategies that target inflammatory tissues (Zhang et al., 2019) (Gao et al., 2019). In this work, the anti-inflammatory effects of HPD on macrophages and lungs of LPS-challenged mice was improved by using the nasal NP-based drug delivery system. In summary, our data provide strong evidence that nasal NP-delivery of HPD protects against CSSassociated ALI and that the nasal NP delivery system could be used to enhance the efficacy of anti-inflammatory agents in the treatment of CSS and ALI/ARDS.

\section{DATA AVAILABILITY STATEMENT}

The raw data supporting the conclusions of this article will be made available by the authors, without undue reservation.

\section{REFERENCES}

Bergsbaken, T., Fink, S. L., and Cookson, B. T. (2009). Pyroptosis: host cell death and inflammation. Nat. Rev. Microbiol., 7 (2), 99-109. doi:10.1038/ nrmicro2070

Bruinsmann, F. A., Pigana, S., Aguirre, T., Dadalt Souto, G., Garrastazu Pereira, G., Bianchera, A., et al. (2019). Chitosan-coated nanoparticles: effect of chitosan molecular weight on nasal transmucosal delivery. Pharmaceutics 11 (2), 86. doi:10.3390/pharmaceutics11020086

Channappanavar, R., and Perlman, S. (2017). Pathogenic human coronavirus infections: causes and consequences of cytokine storm and immunopathology. Semin. Immunopathol., 39 (5), 529-539. doi:10.1007/s00281-017-0629-x

Ciftci, O., Ozcan, C., Kamisli, O., Cetin, A., Basak, N., and Aytac, B. (2015). Hesperidin, a citrus flavonoid, has the ameliorative effects against experimental autoimmune encephalomyelitis (EAE) in a C57BL/J6 mouse model. Neurochem. Res., 40 (6), 1111-1120. doi:10.1007/s11064-015-1571-8

Du, Z. A., Sun, M. N., and Hu, Z. S. (2018). Saikosaponin a ameliorates LPSinduced acute lung injury in mice. Inflammation, 41 (1), 193-198. doi:10.1007/ s10753-017-0677-3

Gao, W., Wang, Y., Xiong, Y., Sun, L., Wang, L., Wang, K., et al. (2019). Sizedependent anti-inflammatory activity of a peptide-gold nanoparticle hybrid in vitro and in a mouse model of acute lung injury. Acta Biomater. 85, 203-217. doi:10.1016/j.actbio.2018.12.046

\section{ETHICS STATEMENT}

The animal study was reviewed and approved by Guangdong Medical University.

\section{AUTHOR CONTRIBUTIONS}

$\mathrm{HJ}, \mathrm{ZZ}$, and XL proposed and supervised the project. HJ, QL, ZM, $\mathrm{HZ}, \mathrm{YW}, \mathrm{XD}, \mathrm{WZ}$, and JP performed the experiments. HJ wrote the paper. CE revised and polished the manuscript. XL provided the funding in this study. All authors have given approval to the final version of the manuscript.

\section{FUNDING}

This work was supported by National Natural Science Foundation of China (No. 81971329, 81671399), Special Funds for the Cultivation of Guangdong College Students'Scientific, Technological Innovation (Climbing Program Special Funds, No. pdjh2019b0224), PhD early development program of Guangdong medical University (B2019012) and "Group-type" Special Supporting Project for Educational Talents in Universities (4SG19057G). CE received an American Heart Association Career Development Award (19CDA34500000).

\section{SUPPLEMENTARY MATERIAL}

The Supplementary Material for this article can be found online at: https://www.frontiersin.org/articles/10.3389/fphar.2020.592238/ full\#supplementary-material.

Garg, U., Chauhan, S., Nagaich, U., and Jain, N. (2019). Current advances in chitosan nanoparticles based drug delivery and targeting. Adv. Pharmaceut. Bull. 9 (2), 195-204. doi:10.15171/apb.2019.023

Gholizadeh, H., Messerotti, E., Pozzoli, M., Cheng, S., Traini, D., Young, P., et al. (2019). Application of a thermosensitive in situ gel of ChitosanBased nasal spray loaded with tranexamic acid for localised treatment of nasal wounds. AAPS PharmSciTech, 20 (7), 299. doi:10.1208/s12249-019-1517-6

Gupta, K. K., Khan, M. A., and Singh, S. K. (2020). Constitutive inflammatory cytokine storm: a major threat to human Health. J. Interferon Cytokine Res. 40 (1), 19-23. doi:10.1089/jir.2019.00851

He, J., Tao, H., Yan, Y., Huang, S. Y., and Xiao, Y. (2020). Molecular mechanism of evolution and human infection with SARS-CoV-2. Viruses 12 (4), 428. doi:10. 3390/v12040428

Hemanth Kumar, B., Dinesh Kumar, B., and Diwan, P. V. (2017). Hesperidin, a citrus flavonoid, protects against l-methionine-induced hyperhomocysteinemia by abrogation of oxidative stress, endothelial dysfunction and neurotoxicity in Wistar rats. Pharm. Biol., 55 (1), 146-155. doi:10.1080/13880209.2016.1231695

Huang, X., Dai, Z., Cai, L., Sun, K., Cho, J., Albertine, K. H., et al. (2016). Endothelial p $110 \gamma \mathrm{PI} 3 \mathrm{~K}$ mediates endothelial regeneration and vascular repair after inflammatory vascular injury. Circulation, 133 (11), 1093-1103. doi:10.1161/CIRCULATIONAHA.115.020918

Jin, H., Pi, J., Zhao, Y., Jiang, J., Li, T., Zeng, X., et al. (2017). EGFR-targeting PLGAPEG nanoparticles as a curcumin delivery system for breast cancer therapy. Nanoscale, 9 (42), 16365-16374. doi:10.1039/c7nr06898k 
Lei, J., Wei, Y., Song, P., Li, Y., Zhang, T., Feng, Q., Xu, G., et al. (2018). Cordycepin inhibits LPS-induced acute lung injury by inhibiting inflammation and oxidative stress. Eur. J. Pharmacol., 818, 110-114. doi:10.1016/j.ejphar.2017.10.029

Li, Y., Kandhare, A. D., Mukherjee, A. A., and Bodhankar, S. L. (2019). Acute and sub-chronic oral toxicity studies of hesperidin isolated from orange peel extract in Sprague Dawley rats. Regul. Toxicol. Pharmacol., 105, 77-85. doi:10.1016/j. yrtph.2019.04.001

Lin, C. W., Tsai, F. J., Tsai, C. H., Lai, C. C., Wan, L., Ho, T. Y., et al. (2005). Anti-SARS coronavirus $3 \mathrm{C}$-like protease effects of Isatis indigotica root and plant-derived phenolic compounds. Antivir. Res., 68 (1), 36-42. doi:10.1016/j.antiviral.2005.07.002

Matthay, M. A., McAuley, D. F., and Ware, L. B. (2017). Clinical trials in acute respiratory distress syndrome: challenges and opportunities. Lancet Respir. Med. 5 (6), 524-534. doi:10.1016/S2213-2600(17)30188-1

Mehta, P., McAuley, D. F., Brown, M., Sanchez, E., Tattersall, R. S., and Manson, J. J., and HLH Across Speciality Collaboration, UK (2020). COVID-19: consider cytokine storm syndromes and immunosuppression. Lancet 395 (10229), 1033-1034. doi:10.1016/S0140

Monfoulet, L. E., Mercier, S., Bayle, D., Tamaian, R., Barber-Chamoux, N., Morand, C., et al. (2017). Curcumin modulates endothelial permeability and monocyte transendothelial migration by affecting endothelial cell dynamics. Free Radic. Biol. Med. 112, 109-120. doi:10.1016/j.freeradbiomed.2017.07.019

Roohbakhsh, A., Parhiz, H., Soltani, F., Rezaee, R., and Iranshahi, M. (2015). Molecular mechanisms behind the biological effects of hesperidin and hesperetin for the prevention of cancer and cardiovascular diseases. Life Sci. 124, 64-74. doi:10.1016/j.lfs.2014.12.030

Russell, C. D., Millar, J. E., and Baillie, J. K. (2020). Clinical evidence does not support corticosteroid treatment for 2019-nCoV lung injury. Lancet 395 (10223), 473-475. doi:10.1016/S0140-6736(20)30317-2

Su, S., Wong, G., Shi, W., Liu, J., Lai, A., Zhou, J., et al. (2016). Epidemiology, genetic recombination, and pathogenesis of coronaviruses. Trends Microbiol., 24 (6), 490-502. doi:10.1016/j.tim.2016.03.003

Sukumar, U. K., Bose, R. J. C., Malhotra, M., Babikir, H. A., Afjei, R., Robinson, E., et al. (2019). Intranasal delivery of targeted polyfunctional gold-iron oxide nanoparticles loaded with therapeutic microRNAs for combined theranostic multimodality imaging and presensitization of glioblastoma to temozolomide. Biomaterials, 218, 119342. doi:10.1016/j.biomaterials.2019.119342
Wang, Y., Li, L., Zhao, W., Dou, Y., An, H., Tao, H., et al. (2018). Targeted therapy of atherosclerosis by a broad-spectrum reactive oxygen species scavenging nanoparticle with intrinsic anti-inflammatory activity. ACS Nano, 12 (9), 8943-8960. doi:10.1021/acsnano.8b02037

Wu, C., Liu, Y., Yang, Y., Zhang, P., Zhong, W., Wang, Y., et al. (2020). Analysis of therapeutic targets for SARS-CoV-2 and discovery of potential drugs by computational methods. Acta Pharm. Sin. B 10 (5), 766-788. doi:10.1016/j. apsb.2020.02.008

Wu, Y. X., He, H. Q., Nie, Y. J., Ding, Y. H., Sun, L., and Qian, F. (2018). Protostemonine effectively attenuates lipopolysaccharide-induced acute lung injury in mice. Acta Pharmacol. Sin. 39 (1), 85-96. doi:10.1038/aps. 2017.131

Yu, X. H., Wang, Y. F., Dai, F. Y., Zhao, J. H., and Li, P. (2019). The protective effects of Berberine and Hesperidin on inflammatory factor-stimulating cardiac fibroblasts. Eur. Rev. Med. Pharmacol. Sci. 23 (12), 5468-5476. doi:10.26355/ eurrev_201906_18216

Zhang, C., Wu, Z., Li, J. W., Zhao, H., and Wang, G. Q. (2020). Cytokine release syndrome in severe COVID-19: interleukin-6 receptor antagonist tocilizumab may be the key to reduce mortality. Int. J. Antimicrob. Agents, 55 (5), 105954. doi:10.1016/j.ijantimicag.2020.105954

Zhang, C. Y., Lin, W., Gao, J., Shi, X., Davaritouchaee, M., Nielsen, A. E., et al. (2019). pH-responsive nanoparticles targeted to lungs for improved therapy of acute lung inflammation/injury. ACS Appl. Mater. Interfaces 11 (18), 16380-16390. doi:10.1021/acsami.9b04051

Conflict of Interest: The authors declare that the research was conducted in the absence of any commercial or financial relationships that could be construed as a potential conflict of interest.

Copyright (c) 2021 Jin, Zhao, Lan, Zhou, Mai, Wang, Ding, Zhang, Pi, Evans and Liu. This is an open-access article distributed under the terms of the Creative Commons Attribution License (CC BY). The use, distribution or reproduction in other forums is permitted, provided the original author(s) and the copyright owner(s) are credited and that the original publication in this journal is cited, in accordance with accepted academic practice. No use, distribution or reproduction is permitted which does not comply with these terms. 